\begin{tabular}{|c|l|}
\hline Title & Minimization of shortfall risk in ajump-diffusion model \\
\hline Author(s) & Nakano, Y. \\
\hline Citation & Hokkaido University Preprint Series in Mathematics, 571, 1-10 \\
\hline Issue Date & 2002-11 \\
\hline DOI & 10.14943/83716 \\
\hline Doc URL & http://hdl.handle.net/2115/69320 \\
\hline Type & bulletin (article) \\
\hline File Information & pre571.pdf \\
\hline
\end{tabular}

Instructions for use 


\section{Minimization of shortfall risk}

in a jump-diffusion model

Yumiharu Nakano

Series \#571. November 2002 


\section{HOKKAIDO UNIVERSITY PREPRINT SERIES IN MATHEMATICS}

\#545 O. Sawada, On time-local solvability of the Navier-Stokes equations in Besov spaces, 30 pages. 2001.

\#546 C. M. Elliott, Y. Giga, and S. Goto, Dynamic boundary conditions for Hamilton-Jacobi equations, 27 pages. 2001.

\#547 Y. Nakano, Minimizing coherent risk measures of shortfall in discrete-time models with cone constraints, 22 pages. 2002 .

\#548 K. tachizawa, A generalization of the Lieb-Thirring inequalities in low dimensions, 13 pages. 2002.

\#549 T. Nakazi, Absolute values and real parts for functions in the Smirnov class, 8 pages. 2002.

\#550 T. Nakazi and T. Watanabe, Properties of a Rubin's orthogonal function which is a linear combination of two inner functions, 9 pages. 2002.

\#551 T. Ohtsuka, A level set method for spiral crystal growth, 24 pages. 2002.

\#552 M.-H. Giga and Y. Giga, Minimal vertical singular diffusion preventing overturning for the Burgers equation, 18 pages. 2002.

\#553 Y. Giga and P. Rybka, Berg's effect, 12 pages. 2002.

\#554 Y. Tonegawa, Domain dependent monotonicity formula for a singular perturbation problem, 14 pages. 2002 .

\#555 S. Izumiya and N. Takeuchi, New special curves and developable surfaces, 10 pages. 2002.

\#556 S. Izumiya, K. Saji and N. Takeuchi, Singularities of line congruences, 17 pages. 2002.

\#557 S. Izumiya, D. Pei and M. C. Romero Fuster, The lightcone Gauss map of a spacelike surface in Minkowski 4-space, 21 pages. 2002.

\#558 S. Izumiya, D. Pei and M. C. Romero Fuster, Umbilicity of spacelike submanifolds of Minkowski space, 14 pages. 2002 .

\#559 S. Izumiya, D. Pei and M. Takahashi, Curves and surfaces in Hyperbolic space, 16 pages. 2002.

\#560 J. Kato, On the uniqueness of nondecaying solutions for the Navier-Stokes equations, 19 pages. 2002.

\#561 M. Jinzenji and T. Sasaki, An Approach to $\mathcal{N}=4 A D E$ gauge Theory on $K 3,29$ pages. 2002

\#562 T.Nakazi and T.Yamamoto, Norms of some singular integral operators on weighted $L^{2}$ spaces, 27 pages. 2002

\#563 A.Harris and Y.Tonegawa, A $\bar{\partial} \partial$-poincaré lemma for forms near an isolated complex singularity, 8 pages. 2002

\#564 M.Takahashi, Bifurcations of ordinary differential equations of Clairaut type, 23 pages.2002

\#565 G.Ishikawa, Classifying singular Legendre curves by contactomorphisms, 17 pages.2002

\#566 G.Ishikawa, Perturbations of Caustics and fronts, 17 pages.2002

\#567 Y.Giga, On regularizing-decay rate estmates for solutions to the Navier-Stokes initial value problem, 12 pages. 2002

\#568 T.Miyao, Strongry supercommuting serf-adjoint operators, 34 pages.2002

\#569 Jun-Muk Hwang and K.Yamaguchi, Characterization of Hermitian symmetric spaces by fundamental forms, 10 pages. 2002

\#570 H.Ishii and T.Mikami, Convexified Gauss curvature flow of bounded open sets in an anisotropic external field: a stochastic apporoximation and PDE, 37 pages 2002 


\title{
Minimization of shortfall risk in a jump-diffusion model
}

\author{
Yumiharu Nakano \\ Division of Mathematics, Graduate School of Science \\ Hokkaido University, Sapporo 060-0810, Japan \\ E-mail: nakano_y@math.sci.hokudai.ac.jp
}

November 8, 2002

\begin{abstract}
We consider a contingent claim in a jump-diffusion model of complete market. Given initial wealth less than the replicating cost, we explicitly solve the problem of minimizing the expected value of hedging loss weighted by power functions. We show that the optimal portfolio is the difference between the perfect hedging portfolio of the contingent claim and the optimal portfolio of a utility minimization problem. We also give an explicit formula for the value function. These results hold for every European-type contingent claim.
\end{abstract}

Key words: shortfall risk, jump-diffusion model.

\section{Introduction}

In this paper, we consider a frictionless, complete financial market consisting of one riskless bond and two risky assets $S_{i}, i=1,2$, that are traded up to a finite time horizon $T$. We suppose that the dynamics of $S_{i}$ are described by jump-diffusion processes. Given a contingent claim $H$ and an initial wealth $x$, we study the following optimization problem:

$$
V(x)=\inf _{\pi \in \mathcal{A}} E\left[\ell_{p}\left(\left(H-X^{x, \pi}(T)\right)_{+}\right)\right],
$$

where $\ell_{p}(x)$ is the power function $x^{p} / p$ with $p>1, X^{x, \pi}$ is the wealth process, and $\mathcal{A}$ is a set of admissible portfolios.

To explain the problem (1), we consider an investor with initial wealth $x$. If $x$ is greater than the replication cost of $H$, say $x_{H}$, then the investor can hedge the contingent claim $H$ without risk, by the completeness of the market. However, if $x$ is strictly less than $x_{H}$, he/she faces the possibility of shortfall, i.e., for any portfolio $\pi$, the shortfall $\left(H-X^{x, \pi}(T)\right)_{+}$may be positive. In this 
situation, one method of hedging $H$ is to follow a portfolio that minimizes the shortfall risk $E\left[\ell_{p}\left(\left(H-X^{x, \pi}(T)\right)_{+}\right)\right]$.

For work related to the problem (1), see, e.g., Föllmer and Leukert [FL] and Pham [P]. See also Nakano [N1] and [N2]. In [FL], general semimartingale models and general loss functions are considered. They impose the nonnegativity constraint on the wealth processes, and use the arguments involving NeymanPearson-type lemmas. This setting is crucial in solving their problem. In this paper, however, instead of the nonnegativity constraint, we impose only an integrability condition on the wealth processes (see Definition 2 below). Thus, our setting is similar to that of $[\mathrm{P}]$, except that we work in a jump-diffusion model of continuous-time markets. By requiring only the integrability condition on the wealth processes, we can obtain not only the optimal terminal wealth but also the optimal portfolio explicitly.

In Section 2, we explain the model and state the precise formulation of our problem. We then show that we can separate the problem into two problems, that is, the perfect hedging problem of $H$ and the utility minimization problem

$$
J\left(x_{H}-x\right)=\inf _{\pi \in \mathcal{A}_{0}\left(x_{H}-x\right)} E\left[\ell_{p}\left(X^{x_{H}-x, \pi}(T)\right)\right],
$$

where $A_{0}\left(x_{H}-x\right)$ is the set of portfolios. We prove that the optimal portfolio of (1) is represented as the difference between the perfect hedging portfolio of $H$ and the optimal portfolio of the problem (2). As in the standard utility maximization problems (cf. Karatzas and Shreve [KS, Chapter 3] and Jeanblanc-Picqué and Pontier [JP]), we can solve the problem (2) by using the martingale method. In our main theorem, we give closed form expressions for the optimal portfolio and the value function $V(x)$. These results hold for every European-type contingent claim, such as, claims that can take negative values and path-dependent options. All the proofs of the results are given in Appendix A.

\section{The model and main results}

We consider a frictionless financial market consisting of one riskless bond $B$ and two risky asset $S_{i}, i=1,2$, that are traded up to a finite time horizon $T$. We suppose that $B$ satisfies the equation

$$
d B(t)=r(t) B(t) d t, \quad B(t)=1 .
$$

We also suppose that $S_{i}, i=1,2$, satisfy the stochastic differential equations

$$
\left\{\begin{array}{lr}
d S_{i}(t)=S_{i}(t-)\left(\mu_{i}(t) d t+\sigma_{i}(t) d W(t)+\gamma_{i}(t) d N(t)\right), \\
S_{i}(0)=s_{i} \in(0, \infty) & (i=1,2),
\end{array}\right.
$$

where $W$ is a one-dimensional standard Brownian motion on a complete probability space $(\Omega, \mathcal{F}, P)$, and the process $N$ is a Poisson process with intensity $\lambda(\cdot)$, which is independent of $W$. The filtration $\left(\mathcal{F}_{t}\right)_{t \geq 0}$ is the $P$-augmentation 
of the natural filtration generated by $W$ and $N$. Then $W$ is a $\left(P, \mathcal{F}_{t}\right)$-Brownian motion, $N$ is a $\left(P, \mathcal{F}_{t}\right)$-Poisson process with intensity $\lambda(\cdot)$, and the process $M(t):=N(t)-\int_{0}^{t} \lambda(s) d s$ is a $P$-martingale.

Assumption 1. For $i=1,2, \lambda, r, \mu_{i}, \sigma_{i}$, and $\gamma_{i}$ are bounded, measurable, deterministic functions on $[0, T]$ that satisfy the following conditions:

(i) $\lambda(t)>0, r(t) \geq 0, \sigma_{i}(t)>0, \gamma_{i}(t)>-1$, and $\gamma_{i}(t) \neq 0$ for $t \in[0, T]$ and $i=1,2$;

(ii) there exists $c_{1} \in(0, \infty)$ such that, for $t \in[0, T]$,

$$
\left|\sigma_{1}(t) \gamma_{2}(t)-\sigma_{2}(t) \gamma_{1}(t)\right| \geq c_{1}
$$

(iii) there exists $c_{2} \in(0, \infty)$ such that, for $t \in[0, T]$,

$$
\frac{\left(\mu_{2}(t)-r(t)\right) \sigma_{1}(t)-\left(\mu_{1}(t)-r(t)\right) \sigma_{2}(t)}{\lambda(t)\left(\sigma_{2}(t) \gamma_{1}(t)-\sigma_{1}(t) \gamma_{2}(t)\right)} \geq c_{2} .
$$

In this paper, we define the investor's wealth process $(X(t))_{0 \leq t \leq T}$ in the standard self-financing way. Thus we assume that $X(\cdot) \equiv X^{x, \pi}(\cdot)$ satisfies

$$
\left\{\begin{aligned}
d X^{x, \pi}(t)= & r(t) X^{x, \pi}(t) d t \\
& +\sum_{i=1}^{2} \pi_{i}(t)\left\{\left(\mu_{i}(t)-r(t)\right) d t+\sigma_{i}(t) d W(t)+\gamma_{i}(t) d N(t)\right\} \\
X^{x, \pi}(0)= & x,
\end{aligned}\right.
$$

where $x \in \mathbf{R}$ is an initial wealth and the portfolio process $\pi(t)=\left(\pi_{1}(t), \pi_{2}(t)\right)$ is an $\mathbf{R}^{2}$-valued $\mathcal{F}_{t}$-predictable process such that all the integrals in $(4)$ are well-defined. The process $\left(\pi_{1}(t), \pi_{2}(t)\right)$ represents the actual amounts of money invested in the risky assets $\left(S_{1}(t), S_{2}(t)\right)$.

Throughout this paper, we fix $p \in(1, \infty)$, and consider the loss function $\ell_{p}$ defined by

$$
\ell_{p}(x)=\frac{x^{p}}{p} \quad(x \geq 0)
$$

We are concerned with the minimization of $E\left[\ell_{p}\left(\left(H-X^{x, \pi}(T)\right)_{+}\right)\right]$over some suitable class of portfolios. To this end, we define the class of admissible portfolios as follows.

Definition 2. A portfolio process $(\pi(t))_{0 \leq t \leq T}$ is said to be admissible if

$$
E\left[\sup _{0 \leq t \leq T}\left|X^{0, \pi}(t)\right|^{p}\right]<\infty .
$$

We write $\mathcal{A}$ for the class of all such $\pi$. 
Put, for $t \in[0, T]$,

$$
\begin{aligned}
\theta(t) & :=\frac{\left(\mu_{2}(t)-r(t)\right) \gamma_{1}(t)-\left(\mu_{1}(t)-r(t)\right) \gamma_{2}(t)}{\sigma_{2}(t) \gamma_{1}(t)-\sigma_{1}(t) \gamma_{2}(t)} \\
\beta(t) & :=\frac{\left(\mu_{2}(t)-r(t)\right) \sigma_{1}(t)-\left(\mu_{1}(t)-r(t)\right) \sigma_{2}(t)}{\lambda(t)\left(\sigma_{2}(t) \gamma_{1}(t)-\sigma_{1}(t) \gamma_{2}(t)\right)} .
\end{aligned}
$$

Then, by Assumption 1, the functions $\theta$ and $\beta$ are bounded, and $\beta$ is positive. Moreover we have

$$
\mu_{i}(t)-r(t)-\sigma_{i}(t) \theta(t)+\lambda \gamma_{i}(t) \beta(t)=0, \quad i=1,2 .
$$

We consider the exponential local martingale

$$
\begin{aligned}
L(t):= & \exp \left(-\int_{0}^{t} \theta(s) d W(s)-\frac{1}{2} \int_{0}^{t}|\theta(s)|^{2} d s\right) \\
& \times \exp \left(\int_{0}^{t} \log \beta(s) d N(s)+\int_{0}^{t} \lambda(s)(1-\beta(s)) d s\right) .
\end{aligned}
$$

From the boundedness of $\theta$ and $\beta$, the process $(L(t))_{0 \leq t \leq T}$ is a strictly positive $P$-martingale, and satisfies, for every $a \in \mathbf{R}$,

$$
E\left[(L(t))^{a}\right]<\infty .
$$

We consider the probability measure $P_{0}$ on $\left(\Omega, \mathcal{F}_{T}\right)$ defined by

$$
\frac{d P_{0}}{d P}=L(T) .
$$

Then, the process

$$
W_{0}(t):=W(t)+\int_{0}^{t} \theta(s) d s \quad(0 \leq t \leq T)
$$

is a $\left(P_{0}, \mathcal{F}_{t}\right)$-Brownian motion, $(N(t))_{0 \leq t \leq T}$ is a $\left(P_{0}, \mathcal{F}_{t}\right)$-Poisson process with intensity $\lambda(t) \beta(t)$, and the process

$$
M_{0}(t):=N(t)-\int_{0}^{t} \lambda(s) \beta(s) d s \quad(0 \leq t \leq T)
$$

is a $P_{0}$-martingale (cf. Brémaud [B]). Using (5), we have, for $i=1,2$,

$$
d \tilde{S}_{i}(t)=\tilde{S}_{i}(t-)\left(\sigma_{i}(t) d W_{0}(t)+\gamma_{i}(t) d M_{0}(t)\right),
$$

where $\tilde{S}_{i}(t)=S_{i}(t) / B(t)$. Thus, $\left(\tilde{S}_{i}(t)\right)_{0 \leq t \leq T}, i=1,2$, are also $P_{0}$-martingales.

In what follows, we use the following notation:

Notation. For a process $(Y(t))_{0 \leq t \leq T}$, we denote by $\tilde{Y}(t)$ the discounted value of $Y(t)$, i.e., $\tilde{Y}(t):=Y(t) / B(t)$. 
For $x \in \mathbf{R}$ and $\pi \in \mathcal{A}$, the discounted wealth process $\tilde{X}^{x, \pi}$ satisfies

$$
\tilde{X}^{x, \pi}(t)=x+\sum_{i=1}^{2} \int_{0}^{t} \tilde{\pi}_{i}(s)\left\{\sigma_{i}(s) d W_{0}(s)+\gamma_{i}(s) d M_{0}(s)\right\}
$$

Thus, $\tilde{X}^{x, \pi}$ is a local $P_{0}$-martingale. However, since $\pi \in \mathcal{A}$, we have from Hölder's inequality and (6) that $E_{0}\left[\sup _{0<t<T}\left|\tilde{X}^{x, \pi}(t)\right|\right]<\infty$, where $E_{0}[\cdot]$ denotes the expectation with respect to $P_{0}$. This implies that $\tilde{X}^{x, \pi}$ is a $P_{0^{-}}$ martingale.

Now, the market above is complete in the following sense:

Proposition 3. Let $H \in L^{p+\varepsilon}\left(\Omega, \mathcal{F}_{T}, P\right)$ for some $\varepsilon>0$, and put

$$
x_{H}:=E_{0}\left[\frac{H}{B(T)}\right] \text {. }
$$

Then there exists unique $\pi_{H} \in \mathcal{A}$ such that, for $t \in[0, T]$,

$$
\tilde{X}_{t}^{x_{H}, \pi_{H}}=E_{0}\left[\frac{H}{B(T)} \mid \mathcal{F}_{t}\right] \quad \text { a.s. }
$$

Let $H \in L^{p+\varepsilon}\left(\Omega, \mathcal{F}_{T}, P\right)$. We interpret $H$ as the investor's liability. By Proposition 3, starting with initial wealth $x_{H}:=E_{0}[H / B(T)]$, the investor can find the replicating portfolio $\pi \in \mathcal{A}$ for $H$. However, if the initial wealth $x$ is less than $x_{H}$, he/she faces the possibility of shortfall. In such a situation, one is naturally led to the minimization of shortfall in an adequate sense. Thus we consider the following stochastic control problem:

$$
V(x):=\inf _{\pi \in \mathcal{A}} E\left[\ell_{p}\left(\left(H-X^{x, \pi}(T)\right)_{+}\right)\right], \quad x<x_{H} .
$$

By definitions of $H$ and $\mathcal{A}$, we easily find that $V(x)<\infty$.

As we stated in Section 1, we can separate the problem above into two problems, that is, the perfect hedging problem of $H$ and a utility minimization problem. For $z>0$, we denote by $\mathcal{A}_{0}(z)$ the set of portfolio processes $(\pi(t))_{0 \leq t \leq T}$ satisfying

$$
X^{z, \pi}(t) \geq 0 \quad t \in[0, T] \quad \text { a.s. }
$$

and

$$
E\left[\sup _{0 \leq t \leq T}\left|X^{0, \pi}(t)\right|^{p}\right]<\infty
$$

We consider another optimization problem, that is,

$$
J(z):=\inf _{\pi \in \mathcal{A}_{0}(z)} E\left[\ell_{p}\left(X^{z, \pi}(T)\right)\right], \quad z>0 .
$$


Proposition 4. For every $z \in(0, \infty)$, there exists $\pi_{0} \in \mathcal{A}_{0}(z)$ that is optimal for the problem (10).

Define $q \in(0, \infty)$ by $(1 / p)+(1 / q)=1$. Here is the main theorem of this paper.

Theorem 5. (i) Let $\pi_{0}$ be as in Proposition \& with $z=x_{H}-x$ and let $\pi_{H}$ be as in Proposition 3. Then $\pi_{H}-\pi_{0}$ is optimal for the problem (1).

(ii) For $(t, u) \in[0, T] \times(0, \infty)$, let $\left(\Pi_{1}, \Pi_{2}\right)$ be the unique solution to the linear system

$$
\left\{\begin{array}{l}
\sigma_{1}(t) \Pi_{1}(t, u)+\sigma_{2}(t) \Pi_{2}(t, u)=-\frac{\theta(t)}{p-1} u \\
\gamma_{1}(t) \Pi_{1}(t, u)+\gamma_{2}(t) \Pi_{2}(t, u)=(\beta(t))^{q-1} u .
\end{array}\right.
$$

Then the optimal portfolio $\pi_{0} \in \mathcal{A}_{0}(z)$ of the problem (10) is given by $\left(\Pi_{1}\left(t, X^{z, \pi_{0}}(t-)\right), \Pi_{2}\left(t, X^{z, \pi_{0}}(t-)\right)\right)$.

(iii) The value function $V(x)$ in (1) is given by

$$
V(x)=\ell_{p}\left(x_{H}-x\right) \exp \left(-(p-1) \int_{0}^{T} a(s) d s\right) \quad\left(x<x_{H}\right),
$$

where $a(\cdot)$ is defined by

$$
a(s)=-q r(s)+\frac{1}{2} q(q-1) \theta^{2}(s)-\lambda(s)\left((q-1)-q \beta(s)+(\beta(s))^{q}\right) .
$$

(iv) The optimal terminal wealth in (1) is given by

$$
\begin{aligned}
& X^{x, \pi_{H}-\pi_{0}}(T) \\
& =H-\left(x_{H}-x\right)(L(T))^{q-1} \exp \left(-\int_{0}^{T}\left(a(s)+\frac{r(s)}{p-1}\right) d s\right) .
\end{aligned}
$$

By Theorem 5 , the problem (1) is reduced to the perfect hedging problem of $H$. If we can obtain the hedging portfolio $\pi_{H}$, then Theorem 5 implies that we can minimize the shortfall risk by following the portfolio $\pi_{H}-\pi_{0}$.

Remark 6. As in the most utility maximization problems, we can associate the problem (10) with a HJB equation. We define the wealth process $X^{t, z, \pi}$ with initial condition $(t, z) \in[0, T] \times(0, \infty)$ as in (4). We also define the class $\mathcal{A}_{0}(t, z)$ of portfolio processes in a way similar to the definition of $\mathcal{A}_{0}(z)$, and put $J(t, z):=\inf _{\pi \in \mathcal{A}_{0}(t, z)} E\left[\ell_{p}\left(X^{t, z, \pi}(T)\right)\right]$. Then, as in [KS, Chapter 3] and [JP, Proposition 5.1], we can prove that the function $J(t, z)$ satisfies the following HJB equation:

$$
\left\{\begin{array}{l}
\frac{\partial J(t, z)}{\partial t}+\inf _{\pi \in \mathbf{R}^{2}} \mathcal{L} J(t, z)=0 \\
J(T, z)=\ell_{p}(z), \quad(t, z) \in[0, T) \times(0, \infty)
\end{array}\right.
$$


where

$$
\begin{aligned}
\mathcal{L} J(t, z) & =z r(t) \frac{\partial J}{\partial z}(t, z)+\sum_{i=1}^{2}\left(\mu_{i}(t)-r(t)\right) \frac{\partial J}{\partial z}(t, z) \\
& +\frac{1}{2}\left(\sum_{i=1}^{2} \pi_{i} \sigma_{i}(t)\right)^{2} \frac{\partial^{2} J}{\partial z^{2}}(t, z)+\lambda\left\{J\left(t, z+\sum_{i=1}^{2} \pi_{i} \gamma_{i}(t)\right)-J(t, z)\right\} .
\end{aligned}
$$

\section{A Proofs}

\section{A.1 Proof of Proposition 3}

Proof of Proposition 4. Let $H \in L^{p+\varepsilon}\left(\Omega, \mathcal{F}_{T}, P_{0}\right)$, and put $x_{H}:=E_{0}[H / B(T)]$. Then, as in [JP, Proposition 2.1], there exists a portfolio process $\pi_{H}$ such that (9) holds. We can easily show the admissibility of $\pi_{H}$ using the $P_{0}$-martingale property of $\tilde{X}$, Hölder's inequality, (6), and Doob's maximal inequality.

\section{A.2 Proof of Proposition 4}

As in the references on the expected utility maximization such as [KS, Chapter 3 , we use the martingale method.

First we write $I(\cdot)$ for the inverse function of $\ell_{p}^{\prime}(\cdot)$, that is, $I(y)=y^{q-1}$ for $y>0$, where $1 / p+1 / q=1$. Let $U_{p}$ be the minus of the Legendre transform of $\ell_{p}$ :

$$
\begin{aligned}
U_{p}(y) & =-\sup _{x>0}\left(x y-\ell_{p}(x)\right)=\inf _{x>0}\left(\ell_{p}(x)-x y\right) \\
& =\ell_{p}(I(y))-y I(y), \quad y>0 .
\end{aligned}
$$

The infimum in (12) is attained by $x=I(y)$.

For $(t, y) \in[0, T] \times(0, \infty)$, we define the process

$$
\begin{aligned}
Y^{t, y}(s)= & y \exp \left(-\int_{t}^{s} r(u) d u-\int_{t}^{s} \theta(s) d W(u)-\frac{1}{2} \int_{t}^{s}|\theta(s)|^{2} d u\right) \\
& \times \exp \left(\int_{t}^{s} \log \beta(u) d N(u)+\int_{t}^{s}(1-\beta(u)) \lambda d u\right), \quad t \leq s \leq T .
\end{aligned}
$$

Then $Y^{t, y}$ satisfies

$$
\left\{\begin{array}{l}
d Y^{t, y}(s)=Y^{t, y}(s-)(-r(s) d s-\theta(s) d W(s)+(\beta(s)-1) d M(s)), \\
Y^{t, y}(t)=y
\end{array}\right.
$$

We put, for $(t, y) \in[0, T] \times(0, \infty)$,

$$
\mathcal{X}(t, y):=E_{0}\left[e^{-\int_{t}^{T} r(s) d s} I\left(Y^{t, y}(T)\right)\right] .
$$


Then, we easily see that

$$
\mathcal{X}(t, y)=y^{1 /(p-1)} \exp \left(\int_{t}^{T} a(s) d s\right),
$$

where $a(\cdot)$ is given by $(11)$. We write $\mathcal{Y}(t, \cdot)$ for the inverse function of $\mathcal{X}(t, \cdot)$, that is,

$$
\mathcal{Y}(t, z)=z^{p-1} \exp \left(-(p-1) \int_{t}^{T} a(s) d s\right) .
$$

Proof of Proposition 4. By (12), we find that, for $y>0$ and $\pi \in \mathcal{A}_{0}(z)$,

$$
\begin{aligned}
& E\left[\ell_{p}\left(X^{z, \pi}(T)\right)\right] \\
& =E\left[\ell_{p}\left(X^{z, \pi}(T)\right)-Y^{0, y}(T) X^{z, \pi}(T)\right]+E\left[Y^{0, y}(T) X^{z, \pi}(T)\right] \\
& =E\left[\ell_{p}\left(X^{z, \pi}(T)\right)-Y^{0, y}(T) X^{z, \pi}(T)\right]+y z \\
& \geq E\left[U_{p}\left(Y^{0, y}(T)\right)\right]+y z .
\end{aligned}
$$

The equality in (15) holds if and only if

$$
X^{z, \pi}(T)=I\left(Y^{0, y}(T)\right) .
$$

However, from Proposition 3 , there exists $\pi_{0} \in \mathcal{A}_{0}(z)$ such that

$$
\tilde{X}^{z, \pi_{0}}(t)=E_{0}\left[e^{-\int_{0}^{T} r(s) d s} I\left(Y^{0, \mathcal{Y}(0, z)}(T)\right) \mid \mathcal{F}_{t}\right] .
$$

Then (15) implies that $E\left[\ell_{p}\left(X^{z, \pi_{0}}(T)\right)\right]=J(z)$.

\section{A.3 Proof of Theorem 5}

Proof of Theorem 5. We consider the stochastic Legendre transform $U(y, \omega)$ of $-\ell_{p}(H(\omega)-z)$ : for $y>0$,

$$
U(y, \omega)=\sup _{-\infty<z \leq H(\omega)}\left\{-\ell_{p}(H(\omega)-z)-y z\right\}=\ell_{q}(y)-y H(\omega) .
$$

The supremum in (17) is attained by $H(\omega)-I(y)$.

From (9) and (16), we have

$$
X^{x_{H}, \pi_{H}}(T)=H, \quad X^{x_{H}-x, \pi_{0}}(T)=I\left(Y^{0, y}(T)\right)
$$

and

$$
E_{0}\left[e^{-\int_{0}^{T} r(u) d u} I\left(Y^{0, y}(T)\right)\right]=x_{H}-x,
$$

where $y:=\mathcal{Y}\left(0, x_{H}-x\right)$. It follows that

$$
X^{x, \pi_{H}-\pi_{0}}(T)=X^{x_{H}, \pi_{H}}(T)-X^{x_{H}-x, \pi_{0}}(T)=H-I\left(Y^{0, y}(T)\right) .
$$


Now, by (17), we see that, for every $\pi \in \mathcal{A}$,

$$
\begin{aligned}
& \left(x_{H}-x\right) \mathcal{Y}\left(0, x_{H}-x\right)-E\left[\ell_{q}\left(\mathcal{Y}\left(0, x_{H}-x\right) e^{-\int_{0}^{T} r(u) d u} L(T)\right)\right] \\
& =E\left[Y^{0, y}(T) H-\ell_{q}\left(Y^{0, y}(T)\right)\right]-x \mathcal{Y}\left(0, x_{H}-x\right) \\
& \leq E\left[Y^{0, y}(T) H-\ell_{q}\left(Y^{0, y}(T)\right)-Y^{0, y}(T)\left(H \wedge X^{x, \pi}(T)\right)\right] \\
& \leq E\left[\ell_{p}\left(H-X^{x, \pi}(T) \wedge H\right)\right]=E\left[\ell_{p}\left(\left(H-X^{x, \pi}(T)\right)_{+}\right)\right] .
\end{aligned}
$$

Both equalities hold in the above inequalities if and only if

$$
H \wedge X^{x, \pi}(T)=H-I\left(Y^{0, y}(T)\right) .
$$

However, (18) implies that the portfolio $\pi_{H}-\pi_{0}$ satisfies (19). Therefore,

$$
\begin{aligned}
V(x) & =E\left[\ell_{p}\left(\left(H-X^{x, \pi_{H}-\pi_{0}}(T)\right)_{+}\right)\right] \\
& =\left(x_{H}-x\right) \mathcal{Y}\left(0, x_{H}-x\right)-E\left[\ell_{q}\left(\mathcal{Y}\left(0, x_{H}-x\right) e^{-\int_{0}^{T} r(u) d u} L(T)\right)\right] .
\end{aligned}
$$

Thus Theorem 5 (i) follows.

From (16) and the Markov property of the process $Y^{t, y}$, we have, for $z>0$,

$$
\begin{aligned}
X^{z, \pi_{0}}(t) & =E_{0}\left[e^{-\int_{t}^{T} r(s) d s} I\left(Y^{0, \mathcal{Y}(0, z)}(T)\right) \mid \mathcal{F}_{t}\right] \\
& =\mathcal{X}\left(t, Y^{0, \mathcal{Y}(0, z)}(t)\right),
\end{aligned}
$$

where $\pi_{0}$ is as in Proposition 4 . Thus

$$
Y^{0, \mathcal{Y}(0, z)}(t)=\mathcal{Y}\left(t, X^{z, \pi_{0}}(t)\right) .
$$

Itô formula and (13) imply that

$$
\begin{aligned}
d\left(e^{-\int_{0}^{t} r(s) d s} \mathcal{X}\left(t, Y^{0, y}(t)\right)\right)= & -\theta(t) e^{-\int_{0}^{t} r(s) d s} Y^{0, y}(t) \mathcal{X}_{y}\left(t, Y^{0, y}(t)\right) d W_{0}(t) \\
& +\left\{\mathcal{X}\left(t, \beta(t) Y^{0, y}(t)\right)-\mathcal{X}\left(t, Y^{0, y}(t)\right)\right\} d M_{0}(t) .
\end{aligned}
$$

From this and (21), we see that

$$
\begin{aligned}
\tilde{X}^{z, \pi_{0}}(t)-z & \\
= & -\int_{0}^{t} \theta(s) e^{-\int_{0}^{s} r(u) d u} \frac{\mathcal{Y}\left(s, X^{z, \pi_{0}}(s)\right)}{\mathcal{Y}_{z}\left(s, X^{z, \pi_{0}}(s)\right)} d W_{0}(s) \\
& +\int_{0}^{t} e^{-\int_{0}^{s} r(u) d u}\left\{\mathcal{X}\left(s, \beta(s) \mathcal{Y}\left(s, X^{z, \pi_{0}}(s-)\right)\right)-X^{z, \pi_{0}}(s-)\right\} d M_{0}(s) .
\end{aligned}
$$

Therefore, by (14) we have

$$
\begin{gathered}
-\theta(t) \frac{\mathcal{Y}(t, u)}{\mathcal{Y}_{z}(t, u)}=-\frac{\theta(t)}{p-1} u=\sum_{i=1}^{2} \sigma_{i}(t) \Pi_{i}(t, u), \\
\mathcal{X}(t, \beta(t) \mathcal{Y}(t, u))-u=\beta(t)^{1 /(p-1)} u=\sum_{i=1}^{2} \gamma_{i}(t) \Pi_{i}(t, u) .
\end{gathered}
$$


Thus, by (22), Theorem 5 (ii) follows.

By (14), (20), and easy computation similar to that of (13), we have

$$
V(x)=\ell_{p}\left(x_{H}-x\right) \exp \left(-(p-1) \int_{0}^{T} a(s) d s\right),
$$

which proves Theorem 5 (iii). Finally, Theorem (5) (iv) follows immediately from (18).

\section{References}

[B] P. Brémaud, Point processes and queues: martingale dynamics, Springerverlag, New York, 1981.

[FL] H. Föllmer and P. Leukert, Efficient hedging: cost versus shortfall risk, Finance Stochast., 4 (2000), 117-146.

[JP] M. Jeanblanc-Picqué and M. Pontier, Optimal portfolio for a small investor in a market model with discontinuous prices, Appl. Math. Optim., 22 (1990), 287-310.

[KS] I. Karatzas and S. E. Shreve, Methods of mathematical finance, SpringerVerlag, New York, 1998.

[N1] Y. Nakano, Efficient hedging with coherent risk measure, submitted.

[N2] Y. Nakano, Minimizing coherent risk measures of shortfall in discrete-time models under cone constraints, submitted.

[P] H. Pham, Dynamic $L^{p}$-hedging in discrete time under cone constraints, SIAM J. Control Optim., 38 (2000), 665-682. 